\title{
A STEM Model to Engage Students in Sustainable Science Education through Sports: A Case Study in Qatar
}

\author{
Ruba Ali, Jolly Bhadra, Nitha Siby, Zubair Ahmad, Noora Jabor Al-Thani
}

\section{Abstract}

Sport is a powerful tool to make deeper connections, stimulations, and understanding of STEM (science, technology, engineering, and mathematics) education. Science in sports is a unique program established to present sports as a medium for STEM education. The program included 248 students (112 females and 136 learning workshops where they were challenged to design sports equipment from innovative materials while adhering to specific design criteria. Quantitative and t-test analysis were performed on data collected over five years from 2012 to 2017 of the program to analyze its effectiveness. Along with Research and Development (R\&D) study obtained from pre and post surveys of students, teachers, and facilitators to further analyze participants' behaviors and attitudes.

\section{Introduction/Objectives}

Sports can promote social integration, economic development, develop teamwork capabilities, and improve physical \& mental health. The state of Qatar has been recently brought to the limelight of sports industry as it has grabbed the opportunity to host the most sought-after sports event, the FIFA world cup 2022. The coverage and discussions on the event and the wide-spread enthusiasm for the sports is a well-timed opportunity for the educational community to emphasize the positive impact of sports on students of all ages. Educators believe sports as paramount while providing a wholesome educational experience. The research objective was solely focused on acquainting students with STEM and its relevance in daily life by engineering simple sports products

\section{Method}

The facilitator offers extensive two-hour daily workshops for a week with diverse hands-on activities (refer to Figure 1) to engage the students in experiential learning. The main goal of providing workshops to the students was to acquaint them with enough scientific information to design and construct the sports products- concrete boat, bowling ball, golf ball, and baseball.

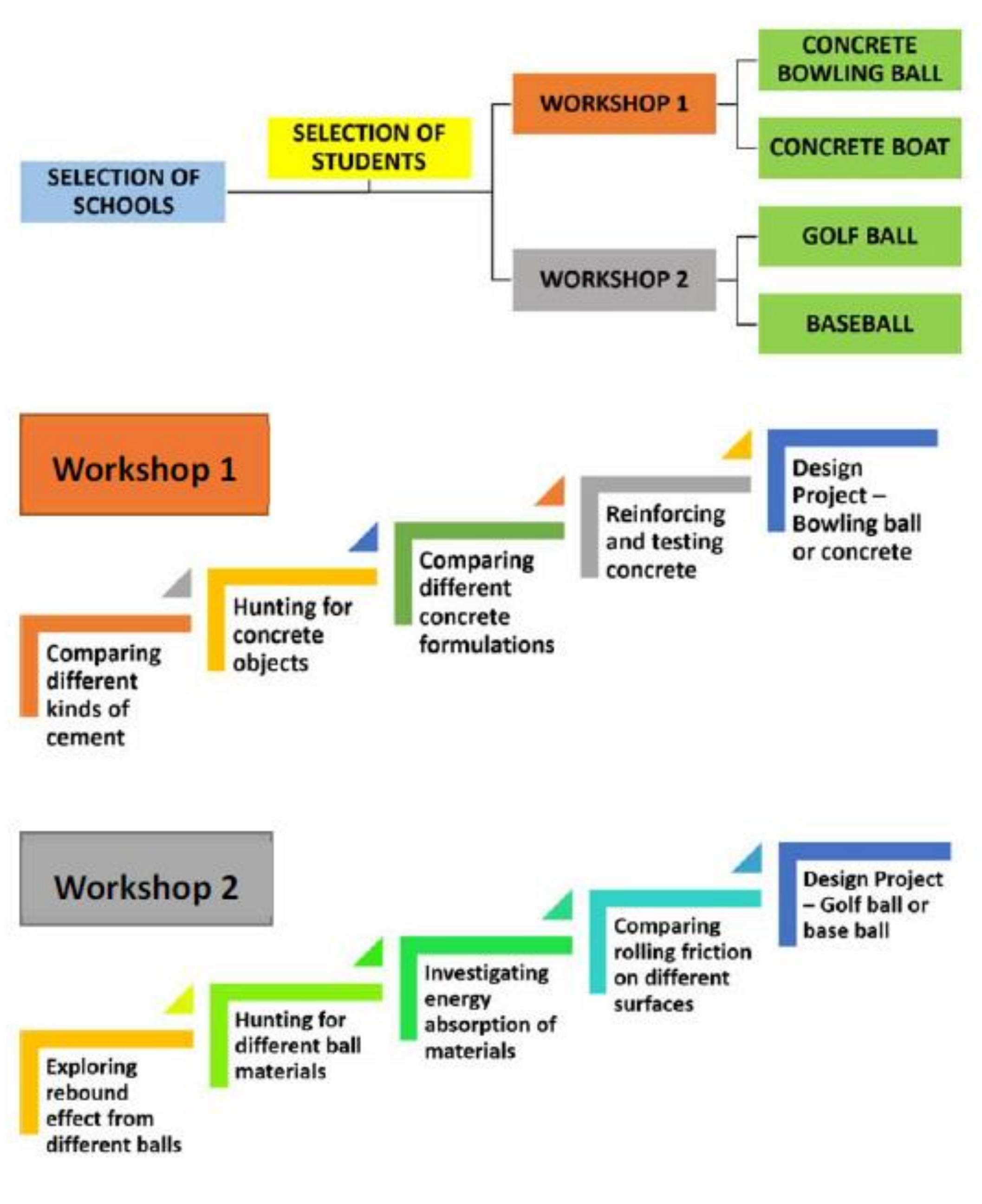

Students adhere to the evaluation criteria, as in Table 1. The sessions were allocated to repeated testing and designing of the product prototypes. In case of failure in creating the product according to the desired criteria, they will start over again or fix the products for positive results.

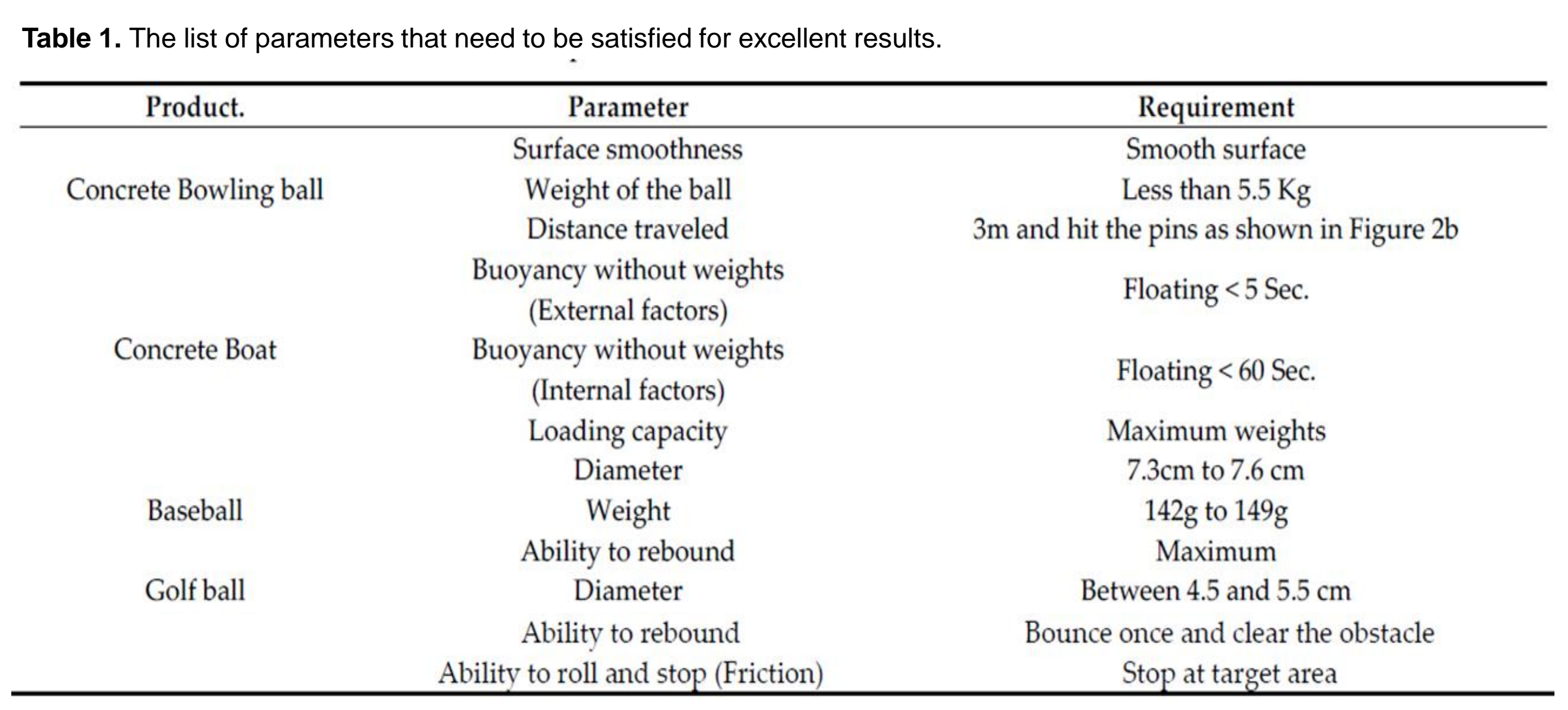

Data Collection Methods

\section{Pre/post -questionnaires}

The students completed a set of study questionnaires to assess their attitude on STEM and its impact in the real world. The questionnaires consist of multiple-choice questions that stem from the student opinion on STEM education, their career interests in scientific fields, and their skills. The students may recommend from four standards of agreement: "agree", "neutral", "disagree," and "do not know". The questionnaires are assessed using standard Monitoring and Evaluation (M\&E) procedures.

\section{Evaluation of project presentation}

After completion, the products were examined by external evaluators from sports facilities, academia and leading economy driving industries. Participants were evaluated on their STEM competencies acquired through the sports product engineering project. They presented their project experience in a PowerPoint presentation, implementing digitalization in the process, and self-mentoring themselves in preparing for the evaluators' queries

\section{Results and Discussion}

The sports-bas their attiludes towards STEM domains and aspirations. The SIS program has positively influenced participant 1. Bowling Ball Design

The participants successfully constructed and designed bowling balls that met different criteria, such as surface smoothness, specific weight, and distance rolled, for the final evaluation.

2. Boat Design

Wesulting boats ported the expected quality of concrete that displayed efficiency in terms of parameters like workability, durability, strength, cost and finishing appearance. Students were successful in presenting a fully functional product.

Students chose optimum materials that contributed to accomplishing their design criteria, specifically balancing the required rebound-ability to cross the obstacle and the desired diameter.

4.. Baseball design

The students were successful in choosing materials that contributed to accomplishing the design criteria, specifically the rebound ability. They combined different materials like rubber, yarn, fabric etc, depending upon their weight and rebound ability to construct a ball structure, looking for the best rebound effect.
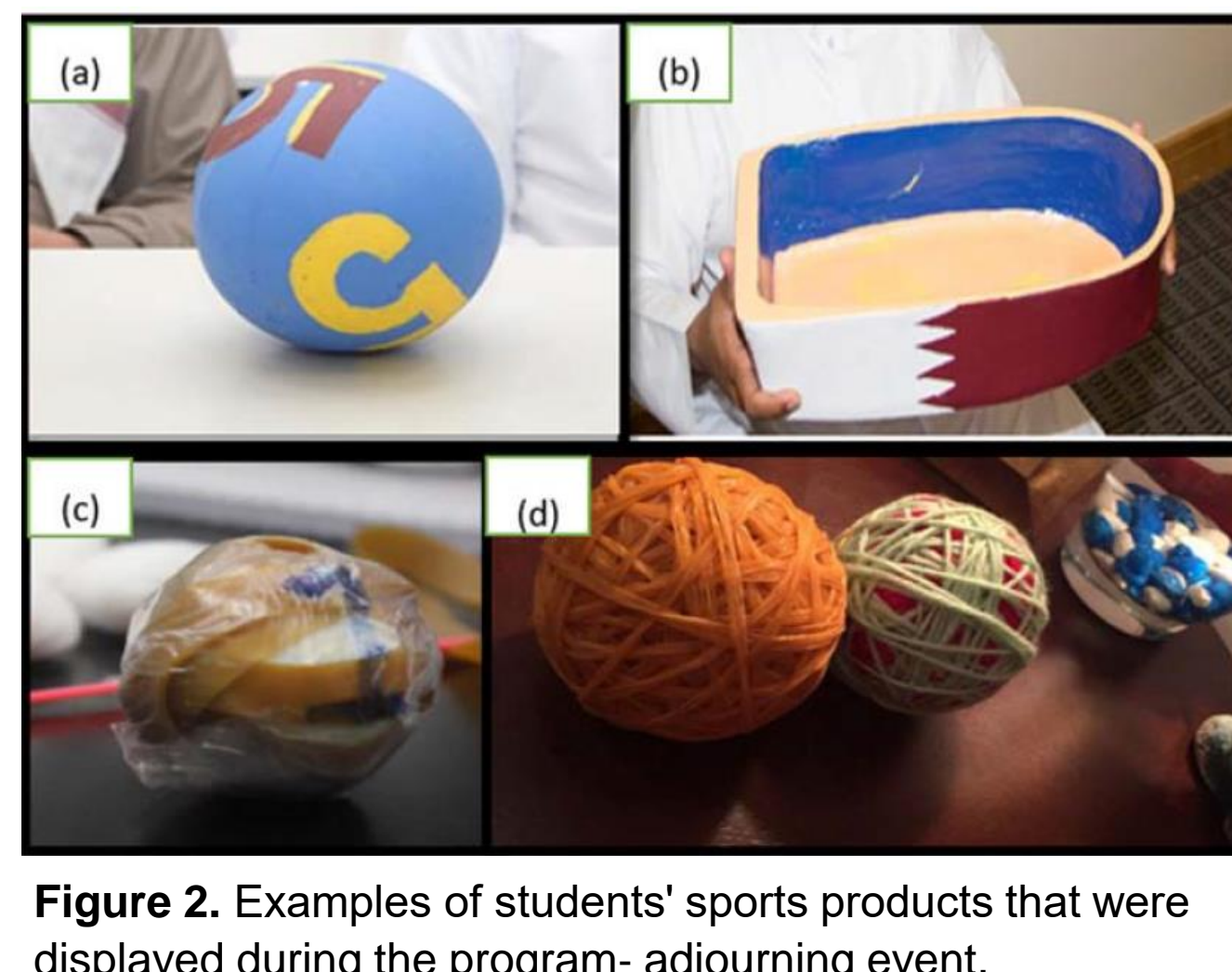

Descriptive Statistical Analysis

Analysis of the study was quantitatively carried out by conducting pre and post workshop surveys. The study clearly manifested the increment in students' interest for interactive STEM curriculum. The analysis also reported a considerable growth in their self-efficacy and innovative skills along with their positive inclination towards STEM, in general and career wise.

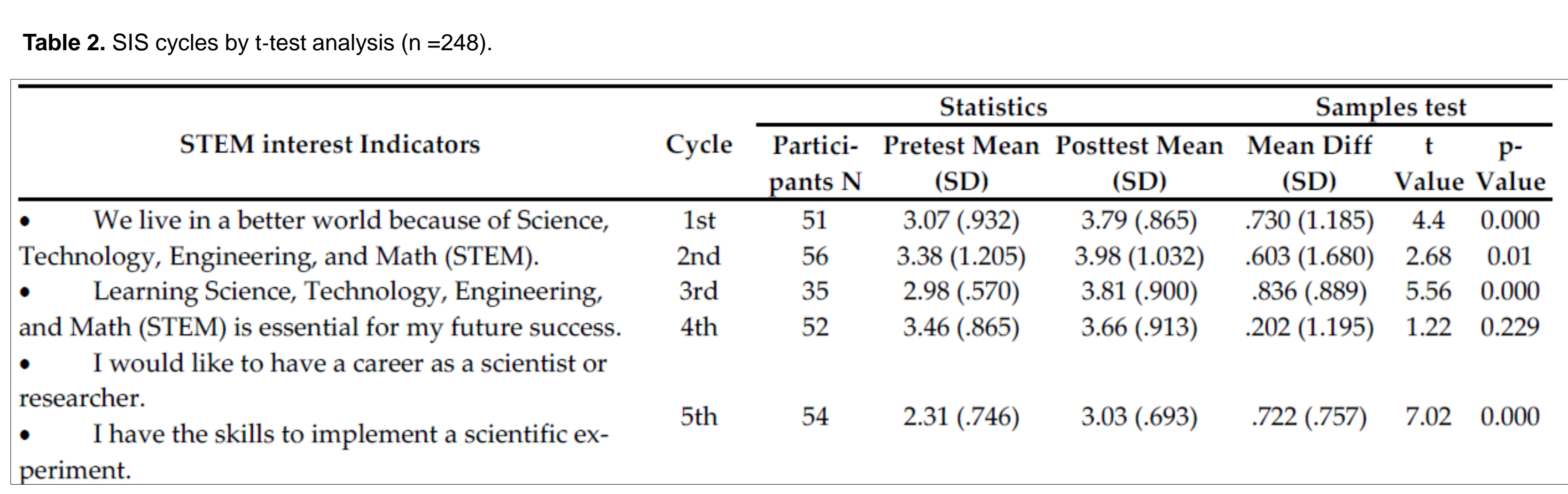

Normalised Mean difference analysis

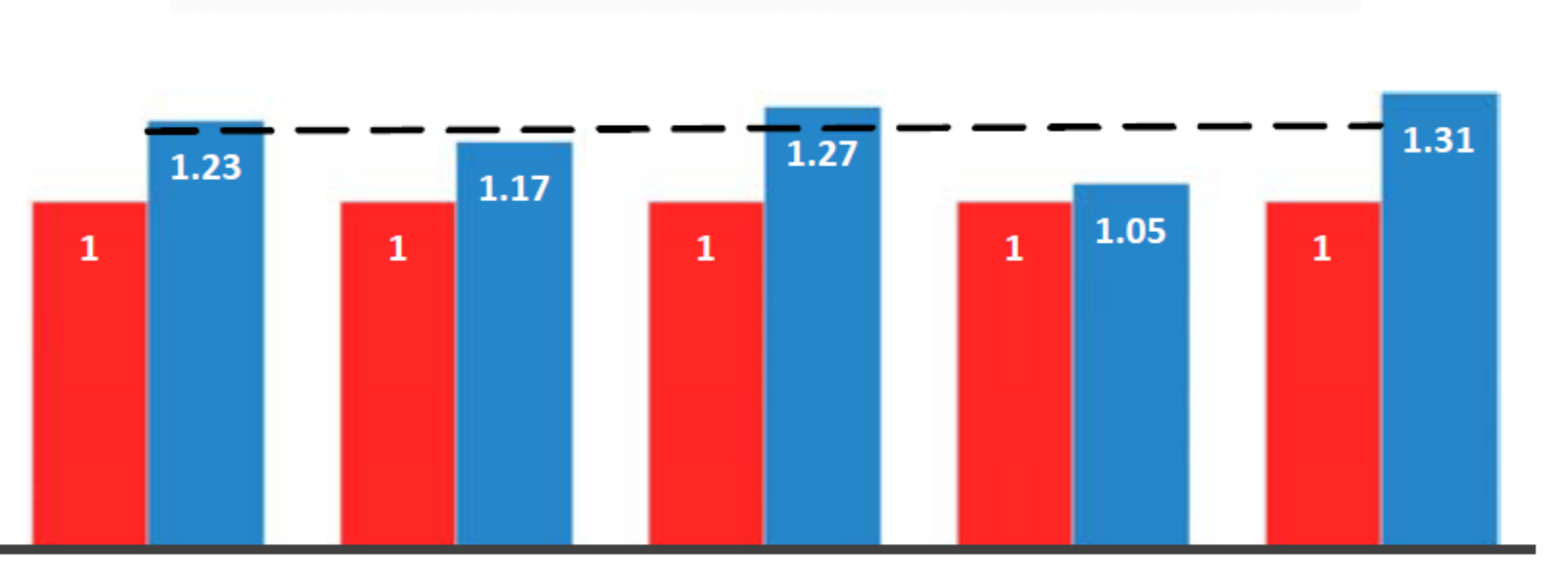

$$
\begin{aligned}
& \text { Figure } 3 \text {. Normalized } \\
& \text { for } 5 \text { cycles }(n=248) \text {. }
\end{aligned}
$$

Demonstration and presentation of design projects

A conference was organized at the end of the program as an adjourning event for two days, displaying and examining the students' final products in the presence of an evaluation panel. As students presented their products to the panel, they displayed their presentation skills along with the communication dispositions. The participants were assessed critically for their organizational ability, research methods implementation, and creative collaboration as they presented their project experience.

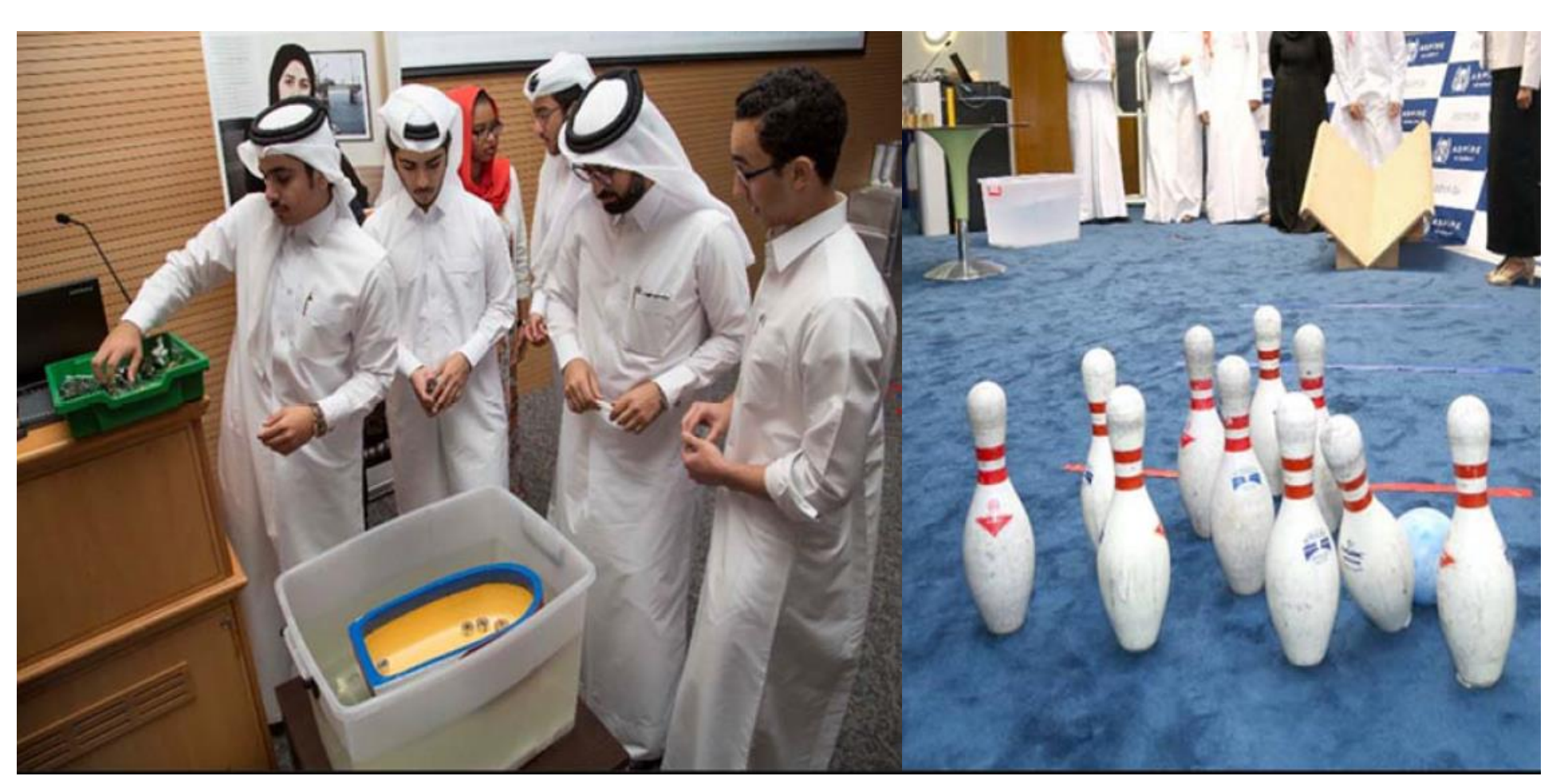

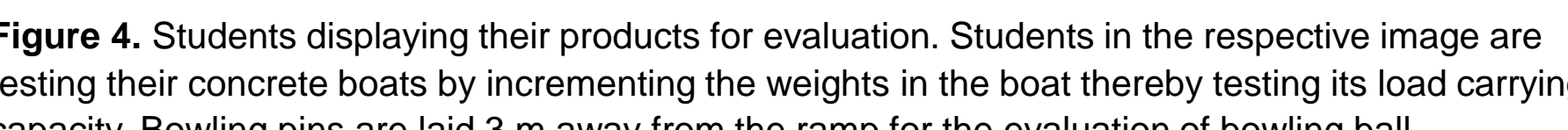

\section{Conclusion}

The implementation of the sports-driven program, 'Science in sport', could enhance high school students' inclination towards STEM fields and careers. Participants self-motivated by sports, bolstered competencies by engaging through engineering design process while developing a sports product during the challenge. The program exploited the students' enthusiasm in sports to participate in an inquiry driven learning approach to experience design and engineering. The program outcome also offers opportunities for student enthusiasm and pride through product achievements.

\section{Acknowledgements}

The authors acknowledge the support from Qatar University Al-Bairaq program to conduct the study.

\section{References}

Craig, E.; Thomas, R.; Hou, C.; Mathur, S. No shortage of talent: How the global market is producing the STEM skills needed for growth. Accenture Institute for High Performance (October, 2011). www. accenture.com/institute 2011 .

Banning, J.; Folkestad, J.E. STEM education related dissertation abstracts: A bounded qualitative meta-study. Journal of Science Education and Technology 2012, 21, 730-741. Aschbacher, P.R.; Ing, M.; Tsai, S.M. Boosting student interest in science. Phi Delta Kappan 2013, 95, 47-51. 15. George, P.S. The Middle School--And Beyond; ERIC: 1992.

Minner, D.D.; Levy, A.J.; Century, J. Inquiry-based science instruction-what is it and does it matter? Results from a research synthesis years 1984 to 2002. Journal of Research in Science Teaching: The Official Journal of the National Association for Research in Science Teaching 2010, 47, 474-496. 